\title{
The role of Monte Carlo within a diagonalization/Monte Carlo scheme
}

\author{
Dean Lee ${ }^{\mathrm{a}}$ \\ ${ }^{a}$ Department of Physics, Univ. of Massachusetts, Amherst, MA 01003
}

\begin{abstract}
We review the method of stochastic error correction which eliminates the truncation error associated with any subspace diagonalization. Monte Carlo sampling is used to compute the contribution of the remaining basis vectors not included in the initial diagonalization. The method is part of a new approach to computational quantum physics which combines both diagonalization and Monte Carlo techniques.
\end{abstract}

\section{INTRODUCTION}

In 11 and [2] a new approach was proposed for finding the low-energy eigenstates of very large or infinite-dimensional quantum Hamiltonians. This proposal combines both diagonalization and Monte Carlo methods, each being used to solve a portion of the problem for which the technique is most efficient. The first part of the proposal is to diagonalize the Hamiltonian restricted to a subspace containing the most important basis vectors for each low energy eigenstate. This may be accomplished either through variational techniques or an $a b$ initio method such as quasi-sparse eigenvector (QSE) diagonalization. The second step is to include the contribution of the remaining basis vectors by Monte Carlo sampling. The use of diagonalization allows one to consider systems with fermion sign oscillations and extract information about wavefunctions and excited states. The use of Monte Carlo provides tools to handle the exponential increase in the number of basis states for large volume systems.

In this brief article we discuss the second half of the diagonalization/Monte Carlo scheme. We discuss several new Monte Carlo techniques known as stochastic error correction (SEC). There are two general varieties of stochastic error correction, methods based on a series expansion and those which are not. The series method starts with an eigenvector of the Hamiltonian restricted to some starting subspace and then includes the contribution of the remaining basis states as terms in an ordered expansion. The idea is to form a perturbative expansion centered around a good non-perturbative starting point.

We also discuss a technique called the stochastic Lanczos method. This method again starts with eigenvectors of a Hamiltonian submatrix. Using these as starting vectors, we define Krylov vectors, $|j\rangle, H|j\rangle, H^{2}|j\rangle \cdots$, similar to standard Lanczos diagonalization. The new ingredient is that matrix elements between Krylov vectors, $\left\langle j^{\prime}\left|H^{n}\right| j\right\rangle$, are computed using matrix diffusion Monte Carlo. Since the method does not rely on a series expansion, it has the advantage that the starting vectors need not be close to the exact eigenvectors.

\section{SERIES METHOD}

Let $|i\rangle$ be the eigenvectors of a Hamiltonian $H$ restricted to some subspace $S$. Let $\left|A_{j}\right\rangle$ be the remaining basis vectors in the full space not contained in $S$. We can represent $H$ as

$$
\left[\begin{array}{ccccc}
\lambda_{1} & 0 & \cdots & \left\langle 1|H| A_{1}\right\rangle & \cdots \\
0 & \lambda_{2} & \cdots & \left\langle 2|H| A_{1}\right\rangle & \cdots \\
\vdots & \vdots & \ddots & \vdots & \cdots \\
\left\langle A_{1}|H| 1\right\rangle & \left\langle A_{1}|H| 2\right\rangle & \cdots & E \cdot \lambda_{A_{1}} & \cdots \\
\vdots & \vdots & \vdots & \vdots & \ddots
\end{array}\right] .
$$

We have used Dirac's bra-ket notation to represent the terms of the matrix. In cases where the basis is non-orthogonal or the Hamiltonian is non-Hermitian, the precise meaning of terms such as $\left\langle A_{1}|H| 1\right\rangle$ is the action of the dual vector 
to $\left|A_{1}\right\rangle$ upon the vector $H|1\rangle$. We have written the diagonal terms for the basis vectors $\left|A_{j}\right\rangle$ with an explicit factor $E$ for reasons to be explained shortly.

Let us assume that $|1\rangle$ is close to some exact eigenvector of $H$ which we denote as $\left|1_{\text {full }}\right\rangle$. More concretely we assume that the components of $\left|1_{\text {full }}\right\rangle$ outside $S$ are small enough so that we can expand in inverse powers of the introduced parameter $E$. In order to simply the expansion we choose to shift the diagonal entries so that $\lambda_{1}=0$.

The series method of stochastic error correction is based on the $E^{-1}$ expansion,

$$
\begin{gathered}
\left|1_{\text {full }}\right\rangle \propto\left[\begin{array}{c}
1 \\
c_{2}^{\prime} E^{-1}+c_{2}^{\prime \prime} E^{-2}+\cdots \\
\vdots \\
c_{A_{1}}^{\prime} E^{-1}+c_{A_{1}}^{\prime \prime} E^{-2}+\cdots \\
\vdots
\end{array}\right], \\
\lambda_{\text {full }}=\lambda_{1}^{\prime} E^{-1}+\lambda_{1}^{\prime \prime} E^{-2} \cdots .
\end{gathered}
$$

It is convenient to choose the normalization of the eigenvector such that the $|1\rangle$ component remains 1. The convergence of the expansion is controlled by the proximity of $|1\rangle$ to $\left|1_{\text {full }}\right\rangle$. If $|1\rangle$ is not at all close to $\left|1_{\text {full }}\right\rangle$ then it will be necessary to use a non-series method such as the stochastic Lanczos method discussed in the next section.

The terms in the series are calculated by Monte Carlo sampling. All that is required is an efficient way of generating random basis vectors $\left|A_{k}\right\rangle$ with known probability rates. Let $P\left(A_{\text {trial }}\right)$ denote the probability of selecting $\left|A_{\text {trial }}\right\rangle$ on a given trial. If for example we are calculating the first order correction to the eigenvalue, then we have

$$
\begin{aligned}
\lambda_{1}^{\prime} & =-\sum_{j} \frac{\left\langle 1|H| A_{j}\right\rangle\left\langle A_{j}|H| 1\right\rangle}{\lambda_{A_{j}}} \\
& =-\lim _{N \rightarrow \infty} \frac{1}{N} \sum_{i=1, \cdots, N} \frac{\left\langle 1|H| A_{\operatorname{trial}(i)}\right\rangle\left\langle A_{\operatorname{trial}(i)}|H| 1\right\rangle}{\lambda_{A_{\text {trial }(i)}} P\left(A_{\operatorname{trial}(i)}\right)} .
\end{aligned}
$$

\section{STOCHASTIC LANCZOS}

We now consider a method called stochastic Lanczos which does not require the starting vectors to be close to exact eigenvectors of $H$. This is essential if the eigenvectors of $H$ are not quasisparse and require extremely large numbers of basis states to represent accurately.

Let $V$ be the full Hilbert space for our system. As in the previous section let $S$ be the subspace over which we have diagonalized $H$ exactly. Let $P_{S}$ be the projection operator for $S$ and let $\lambda_{j}$ and $|j\rangle$ be the eigenvalues and eigenvectors of $H$ restricted to $S$ so that

$$
P_{S} H P_{S}|j\rangle=\lambda_{j}|j\rangle \text {. }
$$

Let $Z$ be an auxiliary subspace, one which contains $S$ but excludes very high-energy states. Let $P_{Z}$ be the projection operator for $Z$. We will choose $Z$ such that $P_{Z} H P_{Z}$ is bounded above. Let $a$ be a real constant which is greater than the midpoint of the minimum and maximum eigenvalues of $P_{Z} H P_{Z}$. The stochastic Lanczos method uses the operators $\left[P_{Z}(H-a) P_{Z}\right]^{n}$ to approximate the low-energy eigenvalues and eigenvectors of $P_{Z} H P_{Z}$. The goal is to diagonalize $H$ in a subspace spanned by vectors

$$
|d, j\rangle=\left[P_{Z}(H-a) P_{Z}\right]^{d}|j\rangle,
$$

for several values of $d$ and $j$. This requires calculating $\left\langle d^{\prime}, j^{\prime} \mid d, j\right\rangle$ and $\left\langle d^{\prime}, j^{\prime}|H| d, j\right\rangle$. If our Hamiltonian matrix is Hermitian, both of these terms can be written in the general form

$$
\left\langle j^{\prime}\left|\left[P_{Z}(H-a) P_{Z}\right]^{n}\right| j\right\rangle .
$$

Therefore it suffices to determine the matrix

$$
A_{n} \equiv P_{S}\left[P_{Z}(H-a) P_{Z}\right]^{n} P_{S} .
$$

For non-orthogonal bases and non-Hermitian Hamiltonians, the only change is that we use vectors

$$
\left[P_{Z}(H-a) P_{Z}\right]^{d}|j\rangle
$$

to generate approximate right eigenvectors of $H$ and vectors in the dual space

$$
\langle j|\left[P_{Z}(H-a) P_{Z}\right]^{d}
$$


to produce approximate left eigenvectors. Adding and subtracting $P_{S}(H-a) P_{S}$, we can rewrite

$A_{n}=P_{S}\left[\begin{array}{c}P_{Z}(H-a) P_{Z}-P_{S}(H-a) P_{S} \\ +P_{S}(H-a) P_{S}\end{array}\right]^{n} P_{S}$.

$A_{n}$ can now be evaluated recursively as

$$
A_{n+1}=B_{n+1}+\sum_{m=0, \cdots, n} B_{m}(H-a) A_{n-m},
$$

where

$$
B_{n}=P_{S}\left[P_{Z}(H-a) P_{Z}-P_{S}(H-a) P_{S}\right]^{n} P_{S} .
$$

The components of $B_{n}$ are computed by matrix diffusion Monte Carlo. Any general matrix product $M^{(1)} M^{(2)} \cdots M^{(n)}$ is a sum of degree $n$ monomials,

$$
\begin{aligned}
& {\left[M^{(1)} M^{(2)} \cdots M^{(n)}\right]_{j k}} \\
& =\sum_{i_{1}, \cdots i_{n-1}} M_{j i_{1}}^{(1)} M_{i_{1} i_{2}}^{(2)} \cdots M_{i_{n-1} k}^{(n)} .
\end{aligned}
$$

We can interpret (14) as a sum over paths through the set of basis vectors of $Z$,

$$
|j\rangle \rightarrow\left|i_{1}\right\rangle \rightarrow \cdots \rightarrow\left|i_{n-1}\right\rangle \rightarrow|k\rangle,
$$

with an associated weight $M_{j i_{1}}^{(1)} M_{i_{1} i_{2}}^{(2)} \cdots M_{i_{n-1} k}^{(n)}$.

\section{Hubbard Model}

As an example of the new diagonalization/Monte Carlo method we consider the twodimensional Hubbard model defined by the Hamiltonian

$$
\begin{aligned}
H & =-t \sum_{<i, j>; \sigma=\uparrow, \downarrow}\left(c_{i \sigma}^{\dagger} c_{j \sigma}+c_{j \sigma}^{\dagger} c_{i \sigma}\right) \\
& +U \sum_{i}\left(c_{i \uparrow}^{\dagger} c_{i \uparrow} c_{i \downarrow}^{\dagger} c_{i \downarrow}\right) .
\end{aligned}
$$

The summation $\langle i, j\rangle$ is over nearest neighbor pairs. $c_{i \sigma}^{\dagger}\left(c_{i \sigma}\right)$ is the creation(annihilation) operator for a spin $\sigma$ electron at site $i$. $t$ is the hopping parameter, and $U$ controls the onsite Coulomb repulsion. The model has attracted considerable attention in recent years due to its possible connection to $d$-wave pairing and stripe correlations in high- $T_{c}$ cuprate superconductors. In spite of its simple form, the computational difficulties associated with finding the ground state of the model are substantial even for small systems. Fermion sign problems render Monte Carlo simulations ineffective for $U$ positive and away from half-filling, and the collective effect of very large numbers of basis Fock states make most diagonalization approaches very difficult. A brief overview of the history and literature pertaining to numerical aspects of the Hubbard model can be found in 3 .

As a test of our methods, we use QSE diagonalization with stochastic error correction to find the ground state energy of the $4 \times 4$ Hubbard model with 5 electrons per spin. The corresponding Hilbert space has about $2 \cdot 10^{7}$ dimensions. For the QSE diagonalization we use momentum Fock states which diagonalize the quadratic part of the Hamiltonian. The Hamiltonian is invariant under the symmetry group generated by reflections about the $x$ and $y$ axes, interchanges between $x$ and $y$, and interchanges between $\downarrow$ and $\uparrow$. We find it convenient to work with symmetrized Fock states. We will compute stochastic error corrections to first order using the series method.

In Table 1 we present results for the ground state energy. We encountered no trouble with the sign problem, and in fact one can easily see that each term in the first order series expression is negative definite. The energies are measured relative to the energy of the Fermi sea at $U=0$. The errors reported are statistical errors associated with the first order SEC calculation. Where available, we compare with the results presented in औ, which we label as Exact, Projector Quantum Monte-Carlo (PQMC), and Stochastic Diagonalization (SD). Stochastic diagonalization is a subspace diagonalization technique similar to QSE but one which uses a different method for selecting the subspace and is based on a variational principle [5]. Although the precise number of basis states used in the SD calculations is 
Table 1

Ground state energy of the $4 \times 4$ Hubbard model

\begin{tabular}{|l|c|l|l|l|l|l|}
\hline Coupling & States & QSE & QSE+SEC & Exact & SD & PQMC \\
\hline \multirow{3}{*}{$U=2 t$} & 100 & -.4797 & $-.50147(5)$ & & & \\
& 500 & -.4945 & $-.50181(3)$ & -.50194 & -.5010 & $-.44(5)$ \\
& 1000 & -.5006 & $-.50198(1)$ & & & \\
\hline \multirow{3}{*}{$U=4 t$} & 100 & -1.620 & $-1.8113(4)$ & & & \\
& 500 & -1.748 & $-1.8242(3)$ & -1.8309 & -1.829 & $-1.8(2)$ \\
& 1000 & -1.800 & $-1.8302(1)$ & & & \\
\hline \multirow{3}{*}{$U=5 t$} & 500 & -2.558 & $-2.7073(4)$ & & & \\
& 1000 & -2.651 & $-2.7208(2)$ & -2.7245 & -2.723 & $-2.9(3)$ \\
& 2000 & -2.685 & $-2.7231(1)$ & & & \\
\hline
\end{tabular}

not listed, we infer from numbers reported for a modified $4 \times 4$ Hubbard system that roughly $10^{5}$ states were used.7.

Apparently QSE diagonalization with SEC handles the $4 \times 4$ system quite well with relatively few states. Much larger systems are being studied using both higher series corrections and stochastic Lanczos techniques [6]. Further examples of both stochastic error correction methods are presented in [2].

\section{ACKNOWLEDGMENTS}

The author thanks all collaborators on the works cited here and the organizers and participants of the Lattice 2000 meeting in Bangalore. Financial support provided by the National Science Foundation.

\section{REFERENCES}

1. D. J. Lee, N. Salwen, D. D. Lee, hepth/0002251.

2. D. Lee, N. Salwen, M. Windoloski, heplat/0010039.

3. D. Scalapino, S. White, cond-mat/0007515.

4. T. Husslein, W. Fettes, I. Morgenstern, condmat/9705026.

5. H. de Raedt, M. Frick, Comp. Phys. Rep. 7 (1992) 1.

6. N. Salwen, D. Lee, work in progress.

${ }^{1}$ The discrete symmetries of the system were not utilized in their calculations. 